\title{
Anal Intraepithelial Neoplasia 1
}

National Cancer Institute

\section{Source}

National Cancer Institute. Anal Intraepithelia/ Neoplasia 1. NCI Thesaurus. Code C157573.

Anal canal or perianal skin intraepithelial neoplasia with mild dysplasia. 